\title{
On-shell methods for the two-loop dilatation operator and finite remainders
}

\author{
Florian Loebbert, ${ }^{a}$ Dhritiman Nandan, ${ }^{a, b}$ Christoph Sieg, ${ }^{a, b}$ Matthias Wilhelm ${ }^{a, b}$ and \\ Gang Yang ${ }^{a}$ \\ ${ }^{a}$ Institut für Physik, Humboldt-Universität zu Berlin, \\ IRIS Gebäude, Zum Großen Windkanal 6, 12489 Berlin, Germany \\ ${ }^{b}$ Institut für Mathematik, Humboldt-Universität zu Berlin, \\ IRIS Gebäude, Zum Großen Windkanal 6, 12489 Berlin, Germany \\ E-mail: loebbert@physik.hu-berlin.de, dhritiman@physik.hu-berlin.de, \\ csieg@physik.hu-berlin.de, mwilhelm@physik.hu-berlin.de, \\ gang ·yang@physik.hu-berlin.de
}

ABSTRACT: We compute the two-loop minimal form factors of all operators in the SU(2) sector of planar $\mathcal{N}=4 \mathrm{SYM}$ theory via on-shell unitarity methods. From the UV divergence of this result, we obtain the two-loop dilatation operator in this sector. Furthermore, we calculate the corresponding finite remainder functions. Since the operators break the supersymmetry, the remainder functions do not have the property of uniform transcendentality. However, the leading transcendentality part turns out to be universal and is identical to the corresponding BPS expression. The remainder functions are shown to satisfy linear relations which can be explained by Ward identities of form factors following from R-symmetry.

KEywords: Scattering Amplitudes, Integrable Field Theories

ARXIV EPRINT: 1504.06323 


\section{Contents}

1 Introduction 1

2 Minimal form factors in the SU(2) sector 4

2.1 Tree-level form factors 4

2.2 One-loop form factors 5

$\begin{array}{lll}2.3 & \text { Two-loop form factors } & 10\end{array}$

3 Two-loop dilatation operator and remainder function $\quad 13$

$\begin{array}{lll}3.1 & \text { Renormalisation constant and dilatation operator } & 15\end{array}$

$\begin{array}{lll}3.2 & \text { Finite remainders } & 15\end{array}$

$\begin{array}{lll}4 & \text { Conclusion and outlook } & 17\end{array}$

$\begin{array}{ll}\text { A Six-point scalar amplitudes } & 19\end{array}$

\section{Introduction}

In the last years, form factors in $\mathcal{N}=4$ super Yang-Mills (SYM) theory have received increasing attention, both at weak coupling [1-17] and at strong coupling [18-20]. Containing both on-shell states and local composite operators, form factors provide a useful bridge between the purely on-shell amplitudes and the off-shell world of correlation functions. In particular, powerful computational methods developed in the context of scattering amplitudes can be applied to form factors and to other important physical quantities via form factors, such as the spectrum of anomalous dimensions of composite operators and their correlation functions. The form factor $\hat{\mathcal{F}}_{\mathcal{O}}$ is defined as the matrix element of a given local operator $\mathcal{O}(x)$ between the vacuum $|0\rangle$ and an on-shell $n$-particle state $\langle 1, \ldots, n|$, i.e.

$$
\hat{\mathcal{F}}_{\mathcal{O}}(1, \ldots, n ; q)=\int \mathrm{d}^{4} x \mathrm{e}^{-i q x}\langle 1, \ldots, n|\mathcal{O}(x)| 0\rangle .
$$

A special class of form factors are the so-called minimal form factors, which contain as many external fields $n$ as there are fields in the operator, and which will be of particular interest for this paper.

Understanding the connection between form factors and the spectral problem of $\mathcal{N}=4$ SYM theory was recently pushed forward in $[16,17]$. In [16], form factors for generic operators were investigated. In particular, it was shown that the complete one-loop dilatation operator [21] can be derived using one-loop minimal form factors, which explains the relation between the one-loop dilatation operator and the four-point scattering amplitude 
derived from symmetry in [22]. ${ }^{1}$ In [17], it was demonstrated that form factors can also be used to calculate anomalous dimensions at two-loop order by investigating the Konishi primary operator. In these studies, on-shell amplitude techniques have played a major role, in particular the (generalised) unitarity method [23-25]. In order to treat general operators, however, an extension of this method is required [17].

Interesting on-shell approaches towards the computation of correlation functions and the dilatation operator were also applied in the following works: see $[10,26]$ for the application of generalised unitarity, [27] for a spacetime version thereof, [28, 29] for twistor techniques and [30] for the application of MHV diagrams.

Computing form factors and correlators of non-protected local gauge-invariant operators requires renormalisation, which in general implies the mixing of these operators. This procedure singles out certain subsectors, which are closed under renormalisation and which transform under subalgebras of the full $\operatorname{PSU}(2,2 \mid 4)$ symmetry [21]. The simplest testing ground for studying the full renormalisation problem of $\mathcal{N}=4$ SYM theory is given by the so-called $\mathrm{SU}(2)$ sector. The operators in this sector are built out of two complex scalar fields $X$ and $Y$ transforming in the fundamental representation of $\mathrm{SU}(2)$, e.g. $X=\phi_{14}$ and $Y=\phi_{24}$. In particular, the single-trace operators are of the form $\mathcal{O}_{\text {bare }}=\operatorname{tr}\left(X^{k_{1}} Y^{k_{2}} X^{k_{3}} Y^{k_{4}} \ldots\right)$, where $k_{j} \in\{0,1,2, \ldots\}$. The renormalised operators of the interacting theory are obtained from these bare operators via the mixing matrix $\mathcal{Z}$ as

$$
\mathcal{O}_{\text {ren }}=\mathcal{Z} \mathcal{O}_{\text {bare }}, \quad \mathcal{Z}=\mathbb{1}+g^{2} \mathcal{Z}^{(1)}+g^{4} \mathcal{Z}^{(2)}+\mathcal{O}\left(g^{6}\right) .
$$

The study of this mixing problem has been of great importance for capturing the novel integrable structures appearing in planar $\mathcal{N}=4$ SYM theory at higher loop orders [31]. At one-loop order, the crucial observation introducing integrability to planar $\mathcal{N}=4 \mathrm{SYM}$ theory was that the anomalous dilatation operator defined as

$$
\delta \mathfrak{D}=-\mu \frac{\mathrm{d}}{\mathrm{d} \mu} \log \mathcal{Z}=2 \varepsilon g^{2} \frac{\partial}{\partial g^{2}} \log \mathcal{Z}=\sum_{\ell=1}^{\infty} g^{2 \ell} \mathfrak{D}^{(\ell)}
$$

takes the form of the integrable Heisenberg spin-chain Hamiltonian within the SU(2) sector $[32] .^{2}$ The central role of the dilatation operator and its interpretation as an (asymptotic) spin-chain Hamiltonian was further emphasized in [33], where the two-loop dilatation operator with $\mathrm{SU}(2)$ symmetry was computed from Feynman diagrams and its three-loop correction was derived under the assumption of integrability. A field-theoretic computation of the latter was later performed in [34]. Making use of integrability, a recursive construction for the asymptotic dilatation operator in the $\mathrm{SU}(2)$ sector is available by now, which allows to compute its operatorial form to high orders in the 't Hooft coupling constant [35, 36].

In this paper, we continue the program of $[16,17]$ and study form factors and the dilatation operator at two-loop order in the full $\mathrm{SU}(2)$ sector. We employ the unitarity

\footnotetext{
${ }^{1}$ Moreover, in [22] symmetry was used to show that all tree-level scattering amplitudes are related to certain contributions to the dilatation operator. The picture of [22] is equivalent to taking cuts of form factors.

${ }^{2}$ In [32], the larger SO(6) sector was actually considered.
} 
method to obtain the complete two-loop form factors in this sector of planar $\mathcal{N}=4 \mathrm{SYM}$ theory. Interestingly, the form factor results satisfy linear relations. It turns out that they can be explained by Ward identities of form factors following from R-symmetry.

Form factors of non-protected operators contain both infrared (IR) divergences, due to soft and collinear virtual momenta, and ultraviolet (UV) divergences. The information of the latter allows us to determine the renormalisation matrix $\mathcal{Z}$, and therefore, the dilatation operator. ${ }^{3}$ In dimensional regularisation, where the four-dimensional theory is continued to $D=4-2 \varepsilon$ dimensions, ${ }^{4}$ all divergences are given by $1 / \varepsilon^{k}$ terms. In order to obtain the dilatation operator, we need to disentangle the IR and UV divergences, which is possible since the IR divergences have a well-understood universal structure [37-40]. Concretely, we will subtract the IR divergences via the BDS ansatz $[41,42]{ }^{5}$ a similar procedure has already been used in $[16,17]$.

For amplitudes, it is well-known that the BDS ansatz does in general not give the full result but allows for a finite remainder function [18], which was first studied for the six-gluon case in [45-47]. For form factors of BPS operators, remainder functions have also been studied in $[8,14]$. In particular, interesting properties associated to the so-called transcendentality were observed, such as the maximal transcendentality principle, which we will review below. In this paper, we will study the remainder functions of form factors of non-protected operators, where new features appear.

Quantities in $\mathcal{N}=4$ SYM theory have shown interesting properties with respect to their transcendentality. Scattering amplitudes and form factors of BPS operators as well as their remainders have uniform transcendentality: ${ }^{6}$ at $\ell$-loop order, they can be expressed as linear combinations of functions and numbers with transcendentality degree $2 \ell$. Furthermore, remarkable relations have been found between the results of $\mathcal{N}=4 \mathrm{SYM}$ theory and QCD. It was first argued in [51] that, for anomalous dimensions of twist-two operators, the $\mathcal{N}=4 \mathrm{SYM}$ theory result is given by the leading transcendental part of the QCD result. This is usually referred to as the maximal transcendentality principle; see also [7, 52-54] for further discussions. While this heuristic relation was observed only for anomalous dimensions, in [8] it was found that the remainder function of certain BPS two-loop form factors matches exactly the leading transcendental part of related two-loop Higgs-to-gluons amplitudes in QCD [55]. This provides a first example where the maximal transcendentality principle is extended from pure numbers to functions which may have non-trivial kinematic dependence. ${ }^{7}$

In this paper, we demonstrate that form factors of non-protected operators show new universality properties regarding their transcendentality. Since the considered operators

\footnotetext{
${ }^{3}$ The anomalous dimensions can then be obtained as eigenvalues of the dilatation operator.

${ }^{4}$ When continuing the spacetime dimension, also the fields have to be continued to $D=4-2 \varepsilon$. This leads to some important subtleties which have been analysed in detail in [17]. These subtleties are, however, absent in the $\mathrm{SU}(2)$ sector.

${ }^{5}$ See also the previous studies of amplitudes in QCD [43, 44].

${ }^{6}$ This is true at least in the cases of lower points or lower loops. There are known examples of amplitudes at sufficient high points in $\mathcal{N}=4 \mathrm{SYM}$ theory which are not given by transcendental functions but elliptic functions [48-50].

${ }^{7}$ An interesting correspondence between the transcendental functions of $\mathcal{N}=4$ SYM theory and QCD was also found for energy-energy correlations [56].
} 
break supersymmetry, the remainder functions are expected not to have the property of uniform transcendentality. However, we find that all contributions of maximal transcendentality are identical to the corresponding results of BPS form factors. This provides further evidence for the universality of the leading transcendental part, which furthermore has a non-trivial kinematic dependence.

This paper is organised as follows. In section 2, we present results for tree-level and one-loop form factors in the $\mathrm{SU}(2)$ sector. This also serves to introduce our conventions and notation. Moreover, we calculate the minimal two-loop form factors of such operators. In section 3, we extract the two-loop dilatation operator and two-loop remainder function from these results. Section 4 contains our conclusions and outlook. We provide simplified expressions for six-point amplitudes appearing in the unitarity calculation in appendix A.

\section{Minimal form factors in the $\mathrm{SU}(2)$ sector}

\subsection{Tree-level form factors}

In this subsection, we summarise some general facts about form factors and give explicit tree-level expressions that are required in the unitarity calculations of the subsequent subsections.

In analogy to amplitudes, we can strip off the gauge-group dependence of the form factors by introducing colour-ordered form factors $\mathcal{F}_{\mathcal{O}}$ :

$$
\hat{\mathcal{F}}_{\mathcal{O}}(1, \ldots, n ; q)=\sum_{\sigma \in \mathbb{S}_{n} / \mathbb{Z}_{n}} \operatorname{tr}\left[\mathrm{T}^{a_{\sigma(1)}} \ldots \mathrm{T}^{a_{\sigma(n)}}\right] \mathcal{F}_{\mathcal{O}}(\sigma(1), \ldots, \sigma(n) ; q)+\text { multi-trace terms }
$$

where $\mathrm{T}^{a}$ with $a=1, \ldots, N_{c}^{2}-1$ are the generators of the gauge group $\mathrm{SU}\left(N_{c}\right)$ and the sum is over all non-cyclic permutations. The multi-trace terms in (2.1) can start to appear at one-loop order but are suppressed in the planar limit, and will not be considered in this paper.

We describe the external on-shell states using Nair's $\mathcal{N}=4$ on-shell superfield [57]:

$$
\Phi(p, \eta)=g_{+}(p)+\eta^{A} \bar{\psi}_{A}(p)+\frac{\eta^{A} \eta^{B}}{2 !} \phi_{A B}(p)+\frac{\epsilon_{A B C D} \eta^{A} \eta^{B} \eta^{C}}{3 !} \psi^{D}(p)+\eta^{1} \eta^{2} \eta^{3} \eta^{4} g_{-}(p)
$$

where $\eta_{A}$ are Graßmann variables that encode the flavour and helicity of the component particles, and $A=1, \ldots, 4$ is the $\mathrm{SU}(4) \mathrm{R}$-symmetry index. In this formalism, we can combine form factors with different external fields into one super form factor. As we will see later, this also makes it easier to study the supersymmetry properties of the form factors.

In this paper, we focus on form factors in the $\mathrm{SU}(2)$ sector. The corresponding singletrace operators involve two complex scalar fields with a common SU(4) index, which are chosen explicitly as $X=\phi_{14}$ and $Y=\phi_{24}$. The tree-level minimal super form factor for the operator $\mathcal{O}=\operatorname{tr}(X X Y X \cdots)$ with $L=n$ fields, for instance, is simply given by

$$
\mathcal{F}_{\mathcal{O}}^{(0)}(1, \ldots, L ; q)=\delta^{4}\left(q-\sum_{i=1}^{L} \lambda_{i} \tilde{\lambda}_{i}\right)\left(\eta_{1}^{1} \eta_{1}^{4} \eta_{2}^{1} \eta_{2}^{4} \eta_{3}^{2} \eta_{3}^{4} \eta_{4}^{1} \eta_{4}^{4} \cdots+\text { cyclic permutations }\right)
$$


In general, the colour-ordered minimal tree-level super form factors of any operator can be obtained from the operator's oscillator representation by replacing the oscillators by spinor helicity variables and multiplying the result by the momentum-conserving delta function [16].

We also need the next-to-minimal tree-level form factors in the two-loop unitarity computation below, which contain one more external field than the minimal ones. They may be computed easily by Feynman diagrams, or obtained from the BPS form factor component expressions, see e.g. [13]. For convenience, we provide some explicit rules that are useful in practice. There are four different cases that can occur. In the first case, a $g_{+}$ can be inserted between two neighbouring positions $i$ and $i+1$. This leads to the following replacement in the colour-ordered minimal tree-level form factor:

$$
\cdots \eta_{i}^{A} \eta_{i}^{B} \eta_{i+1}^{C} \eta_{i+1}^{D} \cdots \longrightarrow \cdots \eta_{i}^{A} \eta_{i}^{B} \frac{\langle i i+2\rangle}{\langle i i+1\rangle\langle i+1 i+2\rangle} \eta_{i+2}^{C} \eta_{i+2}^{D} \cdots
$$

In the second case, a $g_{-}$can be inserted at the same position, leading to

$$
\cdots \eta_{i}^{A} \eta_{i}^{B} \eta_{i+1}^{C} \eta_{i+1}^{D} \cdots \longrightarrow \cdots \eta_{i}^{A} \eta_{i}^{B} \frac{[i i+2]}{[i i+1][i+1 i+2]} \eta_{i+1}^{1} \eta_{i+1}^{2} \eta_{i+1}^{3} \eta_{i+1}^{4} \eta_{i+2}^{C} \eta_{i+2}^{D} \cdots
$$

In the third case, a $\phi_{C D}$ at position $i$ is split into two anti-fermions $\bar{\psi}_{C}$ and $\bar{\psi}_{D}$. This leads to

$$
\cdots \eta_{i-1}^{A} \eta_{i-1}^{B} \eta_{i}^{C} \eta_{i}^{D} \eta_{i+1}^{E} \eta_{i+1}^{F} \cdots \longrightarrow \cdots \eta_{i-1}^{A} \eta_{i-1}^{B} \frac{1}{\langle i i+1\rangle}\left(\eta_{i}^{C} \eta_{i+1}^{D}-\eta_{i}^{D} \eta_{i+1}^{C}\right) \eta_{i+2}^{E} \eta_{i+2}^{F} \cdots
$$

In the fourth case, the $\phi_{C D}$ is split into two fermions $\psi^{C^{\prime}}$ and $\psi^{D^{\prime}}$ with $\epsilon_{C D C^{\prime} D^{\prime}}=1$, leading to

$$
\cdots \eta_{i-1}^{A} \eta_{i-1}^{B} \eta_{i}^{C} \eta_{i}^{D} \eta_{i+1}^{E} \eta_{i+1}^{F} \cdots \longrightarrow \cdots \eta_{i-1}^{A} \eta_{i-1}^{B} \frac{-1}{[i i+1]}\left(\bar{\eta}_{i, C^{\prime}} \bar{\eta}_{i+1, D^{\prime}}-\bar{\eta}_{i, D^{\prime}} \bar{\eta}_{i+1, C^{\prime}}\right) \eta_{i+2}^{E} \eta_{i+2}^{F} \cdots
$$

where $\bar{\eta}_{i, A}=\frac{1}{3 !} \epsilon_{A B C D} \eta_{i}^{B} \eta_{i}^{C} \eta_{i}^{D}$ and the minus sign is related to the order of the $\eta$ 's. The complete next-to-minimal form factor is obtained by summing over all four replacements and all insertion points.

\subsection{One-loop form factors}

In this subsection, we consider the one-loop minimal form factors in the $\mathrm{SU}(2)$ sector and show how to obtain the one-loop dilatation operator from them. This also allows us to introduce our notation and some important concepts that are required for the two-loop case. The results for the one-loop form factors, as well as the recipe to obtain the oneloop dilatation operator, were already given in [16]. Here, a useful new formulation, given in (2.11), is developed, which will be convenient to study the symmetry properties of form factors.

Form factors in the loop expansion can be written in the following form:

$$
\mathcal{F}_{\mathcal{O}}=\left(1+g^{2} \mathcal{I}^{(1)}+g^{4} \mathcal{I}^{(2)}+\ldots\right) \mathcal{F}_{\mathcal{O}}^{(0)}
$$


For operators that are eigenstates under renormalisation, such as BPS operators or the Konishi primary, $\mathcal{I}^{(\ell)}$ is simply the ratio of the $\ell$-loop and tree-level form factor. However, for form factors of operators that renormalise non-diagonally, this is no longer the case, because the loop corrections to vanishing tree-level form factors can be non-vanishing. To overcome this problem, it is necessary to promote $\mathcal{I}^{(\ell)}$ to an operator that acts on the tree-level form factor $\mathcal{F}_{\mathcal{O}}^{(0)}$ and creates a different tree-level form factor from it.

In the planar limit, connected $\ell$-loop interactions can maximally involve $\ell+1$ neighbouring fields in the colour-ordered form factor at a time. Hence, $\mathcal{I}^{(\ell)}$ can be written as an interaction density that is summed over all insertion points. At one-loop order, the maximal interaction range is two, and we can write

$$
\mathcal{I}^{(1)}=\sum_{i=1}^{L} I_{i i+1}^{(1)} .
$$

Here, $L$ denotes the length of the operator $\mathcal{O}, I_{i i+1}^{(1)}$ acts on the external fields $i$ and $i+1$ and cyclic identification $i+L \sim i$ is understood. We depict $I_{i i+1}^{(1)}$ as

$$
I_{i i+1}^{(1)}=I_{i}^{(1)},
$$

where we in general specify only the first field $i$ that is acted on when the range is explicitly specified by the number of occurring legs.

In the $\mathrm{SU}(2)$ sector, the following six range-two interactions are allowed by R-charge conservation: $X X \rightarrow X X, X Y \rightarrow X Y, X Y \rightarrow Y X, Y Y \rightarrow Y Y, Y X \rightarrow Y X$ and $Y X \rightarrow X Y$. It is sufficient to consider the first three, as the last three can be obtained from them by replacing $X \leftrightarrow Y$, which is a symmetry of the theory. We denote the contribution to a given combination of external fields $Z_{A} Z_{B} \rightarrow Z_{C} Z_{D}$ by $\left(I_{i}^{(1)}\right)_{Z_{A} Z_{B}}^{Z_{D}}$, where $Z_{1}=X$, $Z_{2}=Y$ and $A, B, C, D=1,2$. In terms of these matrix elements, the operator $I_{i i+1}^{(1)}$ is explicitly given by

$$
I_{i i+1}^{(1)}=\sum_{A, B, C, D=1}^{2}\left(I_{i}^{(1)}\right)_{Z_{A} Z_{B} Z_{D}} \eta_{i}^{C} \frac{\partial}{\partial \eta_{i}^{A}} \eta_{i+1}^{D} \frac{\partial}{\partial \eta_{i+1}^{B}}
$$

The matrix elements $\left(I_{i}^{(1)}\right)_{Z_{A} Z_{B}}^{Z_{D}}$ can be computed via unitarity. In the one-loop case, we only need to consider the double cut shown in figure 1 . Let us briefly consider the $\left(I_{1}^{(1)}\right)_{X Y}^{Y X}$ case. The cut integrand is given by

$$
\int \mathrm{dLIPS}\left(l_{1}, l_{2}\right) \mathrm{d}^{4} \eta_{l_{1}} \mathrm{~d}^{4} \eta_{l_{2}} \mathcal{F}_{\mathcal{O}}^{(0)}\left(l_{1}^{X}, l_{2}^{Y}, p_{3}, \ldots, p_{L} ; q\right) \mathcal{A}_{4}^{(0)}\left(-l_{2},-l_{1}, p_{1}^{Y}, p_{2}^{X}\right)
$$

where the tree-level form factor is given in (2.3) and the four-point amplitude is given by the standard MHV expression. The labelling of the external legs with $X, Y$ in the tree-level amplitude and form factor means to take the corresponding $\eta$ components; for example, $\mathcal{A}_{4}\left(-l_{2},-l_{1}, p_{1}^{Y}, p_{2}^{X}\right)$ means to take the component of $\mathcal{A}_{4}\left(-l_{2},-l_{1}, p_{1}, p_{2}\right)$ containing the 


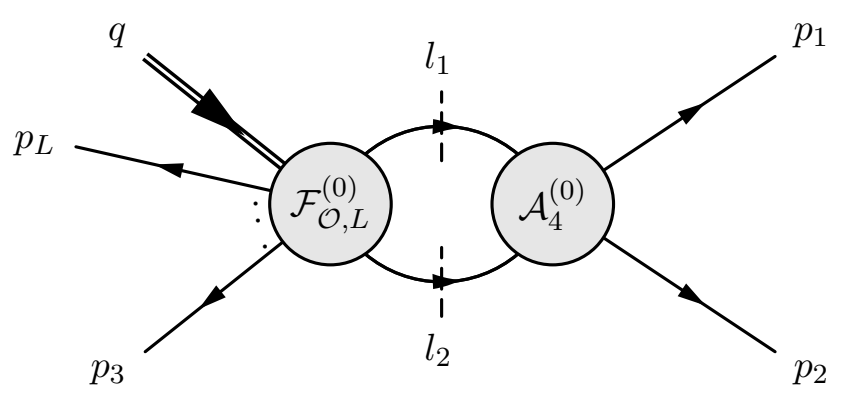

Figure 1. The one-loop $\left(p_{1}+p_{2}\right)^{2}$ double cut.

$\left(\eta_{1}^{2} \eta_{1}^{4}\right)\left(\eta_{2}^{1} \eta_{2}^{4}\right)$ factor. Integrating out the $\eta_{l_{i}}$ variables, the cut integrand is given by ${ }^{8}$

$$
\left.\left(\eta_{1}^{2} \eta_{1}^{4}\right)\left(\eta_{2}^{1} \eta_{2}^{4}\right) \mathcal{F}_{\mathcal{O}}^{(0)}\left(p_{1}^{X}, p_{2}^{Y}, p_{3}, \ldots, p_{L} ; q\right)\right|_{\eta_{1}^{A}=\eta_{2}^{A}=1} \int \mathrm{dLIPS}\left(l_{1}, l_{2}\right)
$$

The variables $\eta_{1}$ and $\eta_{2}$ indicate that the result is not necessarily proportional to the tree-level form factor of the original operator but to the one of the operator in which the corresponding $X$ and $Y$ fields are permuted. The occurring phase space integral is simply the cut of a scalar bubble integral:

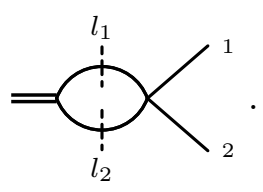

At one-loop level, this cut is sufficient to determine the matrix element $\left(I_{1}^{(1)}\right)_{X Y}^{Y X}$ as the bubble integral. The other matrix elements can be obtained in a similar way. More details of such computations can be found e.g. in [16, 17].

The one-loop results are summarised in table 1 . It is interesting to note that

$$
\left(I_{i}^{(1)}\right)_{X Y}^{X Y}+\left(I_{i}^{(1)}\right)_{X Y}^{Y X}=\left(I_{i}^{(1)}\right)_{X X}^{X X}
$$

This relation is a consequence of the $\mathrm{SU}(2)$ symmetry of the theory. Let us establish a formalism to deal with these symmetries in more detail since it demonstrates the general principle of how symmetries can be used to study form factors.

The PSU $(2,2 \mid 4)$ symmetry of $\mathcal{N}=4$ SYM theory leads to the following Ward identity of form factors:

$$
\sum_{i=1}^{n} \mathfrak{J}_{i}^{A} \mathcal{F}_{\mathcal{O}}(1, \ldots, n ; q)=\mathcal{F}_{\mathfrak{J}^{A} \mathcal{O}}(1, \ldots, n ; q),
$$

${ }^{8}$ Note that

$$
\left.\mathcal{F}_{\mathcal{O}}^{(0)}\left(p_{1}^{X}, p_{2}^{Y}, p_{3}, \ldots, p_{L} ; q\right)\right|_{\eta_{1}^{A}=\eta_{2}^{A}=1}=\left.\frac{\partial^{2}}{\partial \eta_{1}^{1} \partial \eta_{1}^{4}} \frac{\partial^{2}}{\partial \eta_{2}^{2} \partial \eta_{2}^{4}} \mathcal{F}_{\mathcal{O}}^{(0)}\left(p_{1}, \ldots, p_{L} ; q\right)\right|_{\eta_{1}^{A}=\eta_{2}^{A}=0} .
$$




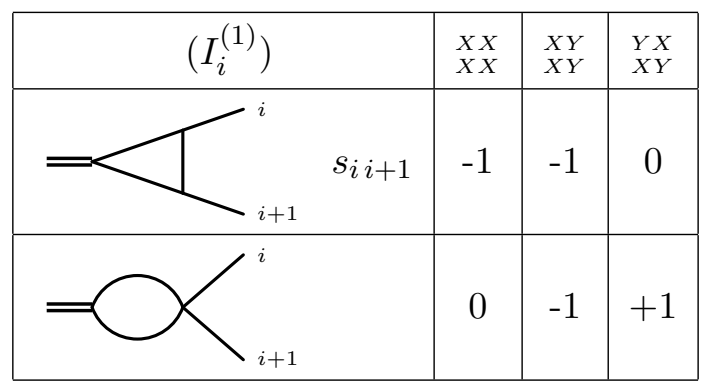

Table 1. Linear combinations of diagrams contributing to the minimal one-loop form factors in the $\mathrm{SU}(2)$ sector.

which holds for any generator $\mathfrak{J}_{i}^{A}$ of $\operatorname{PSU}(2,2 \mid 4)$; see e.g. [4] for a detailed derivation. Let us consider explicitly the generators ${ }^{9}$

$$
\mathfrak{J}_{i}^{1}=\eta_{i}^{1} \frac{\partial}{\partial \eta_{i}^{2}}+\eta_{i}^{2} \frac{\partial}{\partial \eta_{i}^{1}}, \quad \mathfrak{J}_{i}^{2}=-i \eta_{i}^{1} \frac{\partial}{\partial \eta_{i}^{2}}+i \eta_{i}^{2} \frac{\partial}{\partial \eta_{i}^{1}}, \quad \mathfrak{J}_{i}^{3}=\eta_{i}^{1} \frac{\partial}{\partial \eta_{i}^{1}}-\eta_{i}^{2} \frac{\partial}{\partial \eta_{i}^{2}}
$$

of $\mathrm{SU}(2)$. Applying (2.17) to (2.8) for the minimal tree-level and one-loop form factor, we find

$$
\left[\mathfrak{J}^{A}, \mathcal{I}^{(1)}\right]=0,
$$

where $\mathfrak{J}^{A}=\sum_{i=1}^{L} \mathfrak{J}_{i}^{A}$. Inserting (2.11) into (2.19) yields (2.16) as well as similar identities.

The results of table 1 contain the one-mass triangle and bubble integral, for which explicit expressions can be found e.g. in [59]. The one-mass triangle integral is IR divergent and UV finite. The bubble integral, on the other hand, is IR finite but UV divergent. Hence, the IR and UV divergences can be separated immediately.

The IR divergences of the above results match the universal form of one-loop IR divergences [1]:

$$
\begin{aligned}
\left.I_{i i+1}^{(1)}\right|_{\mathrm{IR}} & =-\frac{1}{\varepsilon^{2}}\left(-s_{i i+1}\right)^{-\varepsilon} \mathbb{1}_{i i+1}+\mathcal{O}\left(\varepsilon^{0}\right) \\
& =\left[-\frac{\gamma_{\text {cusp }}^{(1)}}{8 \varepsilon^{2}}-\frac{\mathcal{G}_{0}^{(1)}}{4 \varepsilon}\right]\left(-s_{i i+1}\right)^{-\varepsilon} \mathbb{1}_{i i+1}+\mathcal{O}\left(\varepsilon^{0}\right),
\end{aligned}
$$

where $\gamma_{\text {cusp }}^{(1)}=8$ is the one-loop cusp anomalous dimension and $\mathcal{G}_{0}^{(1)}=0$ is the one-loop collinear anomalous dimension. We have also introduced the identity operator

$$
\mathbb{1}_{i i+1}=\sum_{A, B=1}^{2} \eta_{i}^{A} \frac{\partial}{\partial \eta_{i}^{A}} \eta_{i+1}^{B} \frac{\partial}{\partial \eta_{i+1}^{B}} .
$$

The UV divergences require the renormalisation of the operators. The renormalised operators are defined in terms of the bare operators and the renormalisation constant $\mathcal{Z}$ as shown in (1.2). The renormalised form factor is nothing but the form factor of the

\footnotetext{
${ }^{9}$ In general, the generators of $\operatorname{PSU}(2,2 \mid 4)$ may obtain anomaly contributions, see e.g. [58]. These are, however, absent for $\mathrm{SU}(2)$.
} 
renormalised operator. ${ }^{10}$ Since the form factor is linear in the operator, we can write in the case of the minimal form factor:

$$
\mathcal{F}_{\mathcal{Z} \mathcal{O}}^{(0)}(1, \ldots, L ; q)=\mathcal{Z F}_{\mathcal{O}}^{(0)}(1, \ldots, L ; q)
$$

where, on the right hand side, $\mathcal{Z}$ acts as an operator on the tree-level form factor, similar to $\mathcal{I}^{(\ell)}$ discussed before, cf. (2.8).

At one-loop level, $\mathcal{Z}^{(1)}$ has to render the renormalised one-loop interaction

$$
\underline{\mathcal{I}}^{(1)}=\mathcal{I}^{(1)}+\mathcal{Z}^{(1)}
$$

UV finite. This means that $\mathcal{Z}_{i i+1}^{(1)}$ has to cancel the UV divergence of the bubble integrals occurring in $I_{i i+1}^{(1)}$. The UV divergence of the bubble integral is given by $\frac{1}{\varepsilon} \cdot{ }^{11}$ Accordingly, using the results in table 1, the one-loop renormalisation constant density is given by the matrix elements

$$
\left(\mathcal{Z}_{i}^{(1)}\right)_{X X}^{X X}=0, \quad\left(\mathcal{Z}_{i}^{(1)}\right)_{X Y}^{X Y}=\frac{1}{\varepsilon}, \quad\left(\mathcal{Z}_{i}^{(1)}\right)_{X Y}^{Y X}=-\frac{1}{\varepsilon} .
$$

It can be written in the compact operatorial form

$$
\mathcal{Z}_{i+1}=\mathcal{Z}_{i}^{(1)}=\frac{1}{\varepsilon}(\mathbb{1}-\mathbb{P})_{i i+1},
$$

where $\mathbb{1}$ is the identity operator $(2.21)$ and

$$
\mathbb{P}_{i i+1}=\sum_{A, B=1}^{2} \eta_{i}^{B} \frac{\partial}{\partial \eta_{i}^{A}} \eta_{i+1}^{A} \frac{\partial}{\partial \eta_{i+1}^{B}}
$$

denotes the permutation operator.

In analogy to the renormalisation constant, we can also write the dilatation operator as an operator acting on the minimal tree-level form factor. Applying (1.3) to (2.24), we find the one-loop dilatation operator density

$$
\left(\mathfrak{D}_{i}^{(1)}\right)_{X X}^{X X}=0, \quad\left(\mathfrak{D}_{i}^{(1)}\right)_{X Y}^{X Y}=2, \quad\left(\mathfrak{D}_{i}^{(1)}\right)_{X Y}^{Y X}=-2 .
$$

These expressions can be combined into the well-known form [32]

$$
\mathfrak{D}_{i i+1}^{(1)}=2(\mathbb{1}-\mathbb{P})_{i i+1}
$$

Let us now proceed to two-loop order.

\footnotetext{
${ }^{10}$ This statement relies on the finiteness of $\mathcal{N}=4 \mathrm{SYM}$ theory and on a formulation in which also wave-function renormalisation is absent.

${ }^{11}$ We use a modified minimal subtraction scheme with effective planar coupling constant $g^{2}=$ $\left(4 \pi \mathrm{e}^{-\gamma_{\mathrm{E}}}\right)^{\varepsilon} \frac{g_{\mathrm{YM}}^{2} N_{c}}{(4 \pi)^{2}}$.
} 


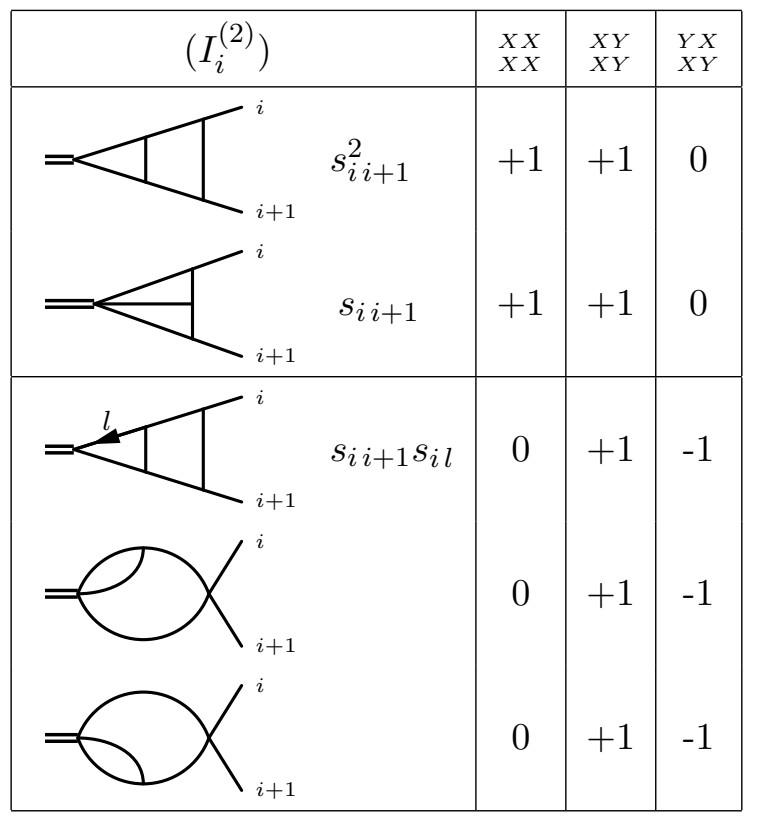

Table 2. Linear combinations of diagrams of range two contributing to the minimal two-loop form factors in the $\mathrm{SU}(2)$ sector. Terms between horizontal lines always occur in fixed combinations.

\subsection{Two-loop form factors}

In the two-loop case, the range of connected interactions can be either two or three. Furthermore, two disconnected one-loop interactions can occur at two-loop level. In total, we can introduce the two-loop operator $\mathcal{I}^{(2)}$ similar to the one-loop case as

$$
\mathcal{I}^{(2)}=\sum_{i=1}^{L}\left(I_{i i+1 i+2}^{(2)}+I_{i i+1}^{(2)}+\frac{1}{2} \sum_{j=i+2}^{L+i-2} I_{i i+1}^{(1)} I_{j j+1}^{(1)}\right),
$$

where the last term accounts for the insertion of two one-loop interactions $I_{i i+1}^{(1)}$ at nonoverlapping positions. The two-loop interactions $I_{i i+1}^{(2)}$ and $I_{i i+1 i+2}^{(2)}$ are given by

$$
\begin{aligned}
I_{i i+1}^{(2)} & =I_{i}^{(2)}=\sum_{A, B, C, D=1}^{2}\left(I_{i}^{(2)}\right){ }_{Z_{A} Z_{B}}^{Z_{C} Z_{D}} \eta_{i}^{C} \frac{\partial}{\partial \eta_{i}^{A}} \eta_{i+1}^{D} \frac{\partial}{\partial \eta_{i+1}^{B}} \\
I_{i i+1 i+2}^{(2)} & =\sum_{A, B, C, D, E, F=1}^{2}\left(I_{i}^{(2)}\right)_{Z_{A} Z_{B} Z_{B} Z_{C}}^{Z_{F}} \eta_{i}^{D} \frac{\partial}{\partial \eta_{i}^{A}} \eta_{i+1}^{E} \frac{\partial}{\partial \eta_{i+1}^{B}} \eta_{i+2}^{F} \frac{\partial}{\partial \eta_{i+2}^{C}} .
\end{aligned}
$$

For interaction range two, three distinct cases occur: $X X \rightarrow X X, X Y \rightarrow X Y$ and $X Y \rightarrow Y X$. We collect our results for the corresponding matrix elements in table 2 and table 3. The matrix elements $\left(I_{i}^{(2)}\right)_{X X}^{X X}$ and $\left(I_{i}^{(2)}\right)_{X X X}^{X X X}$ occur in the BPS case and were computed in [14] using the unitarity method. The other matrix elements can be calculated in a similar but slightly more involved computation. Let us give a brief account of this computation.

For interaction range three, six distinct cases occur: $X X X \rightarrow X X X, X X Y \rightarrow X X Y$, $X Y X \rightarrow X Y X, X X Y \rightarrow X Y X, X Y X \rightarrow X X Y$ and $X X Y \rightarrow Y X X$. The remaining 


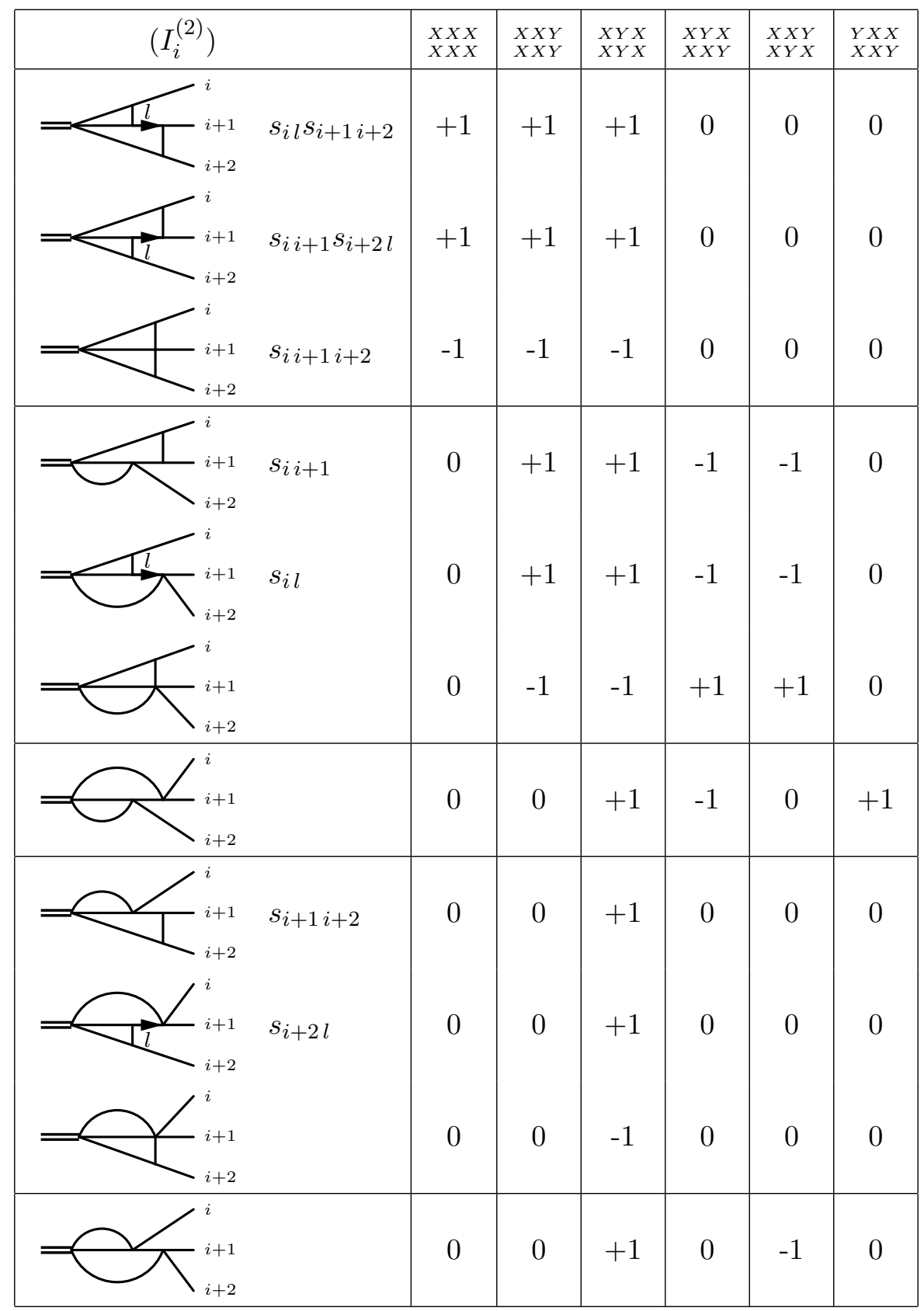

Table 3. Linear combinations of integrals of range three contributing to the minimal two-loop form factors in the $\mathrm{SU}(2)$ sector. The integrals are grouped such that those between two horizontal lines are always occurring in the same combination. The second and fourth group as well as the third and fifth group are related by parity.

combinations can be obtained from these cases by exchanging $X \leftrightarrow Y$ and by using parity, i.e. reverting the order of the fields.

The cuts that have to be considered are depicted in figures $2 \mathrm{a}, 2 \mathrm{~b}, 2 \mathrm{c}$ and $2 \mathrm{~d}$. The treelevel next-to-minimal and one-loop minimal form factors, which occur as building blocks, are given in subsections 2.1 and 2.2, respectively. The required tree-level and one-loop 


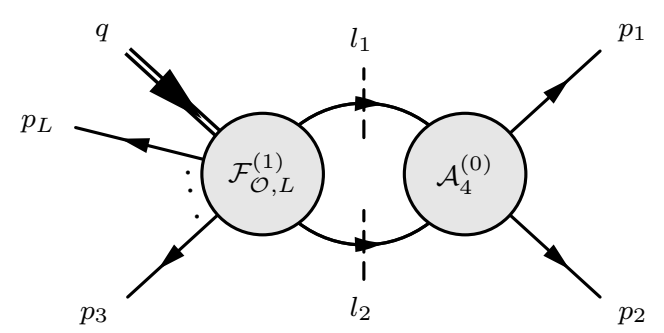

(a) One two-loop $\left(p_{1}+p_{2}\right)^{2}$ double cut.

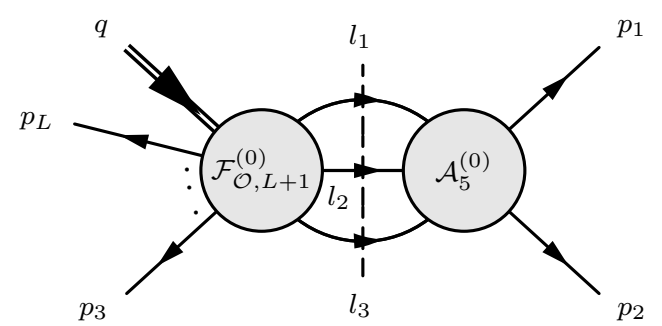

(c) The two-loop $\left(p_{1}+p_{2}\right)^{2}$ triple cut.

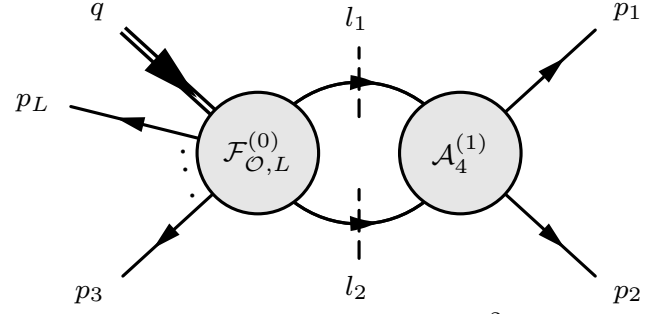

(b) Another two-loop $\left(p_{1}+p_{2}\right)^{2}$ double cut.

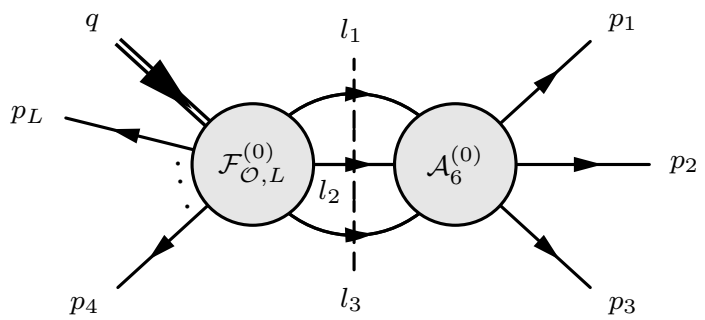

(d) The two-loop $\left(p_{1}+p_{2}+p_{3}\right)^{2}$ triple cut.

Figure 2. Unitary cuts of the minimal two-loop form factor.

amplitudes are standard. A particularly interesting cut is the triple cut shown in figure $2 \mathrm{~d}$, which involves the tree-level next-to-MHV six-point scalar amplitudes. As given explicitly in appendix A, these scalar amplitudes take a simple form in terms of Mandelstam variables. Let us consider for example the $\left(I_{i}^{(2)}\right)_{X X Y}^{Y X X}$ case. The cut integrand is given by the product of the minimal tree-level form factor and the six-point tree-level amplitude:

$$
\int \mathrm{d} \operatorname{LIPS}\left(l_{1}, l_{2}, l_{3}\right) \prod_{i=1}^{3} \mathrm{~d}^{4} \eta_{l_{i}} \mathcal{F}_{\mathcal{O}}^{(0)}\left(l_{1}^{X}, l_{2}^{X}, l_{3}^{Y}, p_{4}, \ldots, p_{L} ; q\right) \mathcal{A}_{6}\left(-l_{3},-l_{2},-l_{1}, p_{1}^{Y}, p_{2}^{X}, p_{3}^{X}\right) .
$$

After integrating out the $\eta_{l_{i}}$ variables, we obtain

$$
\begin{aligned}
& \left.\mathcal{F}_{\mathcal{O}}^{(0)}\left(p_{1}^{X}, p_{2}^{X}, p_{3}^{Y}, \ldots, p_{L} ; q\right)\right|_{\eta_{1}^{A}=\eta_{2}^{A}=\eta_{3}^{A}=1}\left(\eta_{1}^{2} \eta_{1}^{4}\right)\left(\eta_{2}^{1} \eta_{2}^{4}\right)\left(\eta_{3}^{1} \eta_{3}^{4}\right) \\
& \quad \times \int \operatorname{dLIPS}\left(l_{1}, l_{2}, l_{3}\right) \frac{1}{\left(-l_{1}+p_{1}+p_{2}\right)^{2}},
\end{aligned}
$$

where we have used (A.2) for $\left(A^{(0)}\right)_{X X Y}^{Y X X}{ }^{12}$ The phase space integral corresponds to the triple-cut loop integral

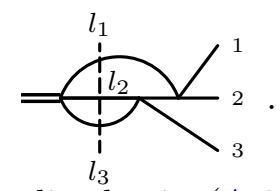

Similarly, each term in the other amplitudes in (A.2) is mapped to one graph in table 3 via the triple cut.

Looking at table 2 and table 3 and using the parity transformation, we observe some linear identities, e.g.

$$
\left(I_{i}^{(2)}\right)_{X Y}^{X Y}+\left(I_{i}^{(2)}\right)_{X Y}^{Y X}=\left(I_{i}^{(2)}\right)_{X X}^{X X}
$$

\footnotetext{
${ }^{12}$ Note that there is a minus sign from the $\eta_{l_{i}}$ integration which cancels the sign in $\left(A^{(0)}\right)_{X X Y}^{Y X X}$.
} 
and

$$
\begin{aligned}
& \left(I_{i}^{(2)}\right)_{X X Y}^{Y X X}+\left(I_{i}^{(2)}\right)_{X X Y}^{X Y X}+\left(I_{i}^{(2)}\right)_{X X Y}^{X X Y}=\left(I_{i}^{(2)}\right)_{X X X}^{X X X}, \\
& \left(I_{i}^{(2)}\right)_{X Y X}^{X Y X}+\left(I_{i}^{(2)}\right)_{X Y X}^{Y X X}+\left(I_{i}^{(2)}\right)_{X Y X}^{X X Y}=\left(I_{i}^{(2)}\right)_{X X X}^{X X X}, \\
& \left(I_{i}^{(2)}\right)_{X X Y}^{X Y X}+\left(I_{i}^{(2)}\right)_{X X Y}^{Y X X}=\left(I_{i}^{(2)}\right)_{X Y X}^{X X Y}+\left(I_{i}^{(2)}\right)_{Y X X}^{X X Y} .
\end{aligned}
$$

Similar to the one-loop case, all these identities are a consequence of SU(2) invariance and follow from the Ward identity (2.17), which at two-loop order yields

$$
\left[\mathfrak{J}^{A}, \mathcal{I}^{(2)}\right]=0 .
$$

Given the full integrand of the two-loop form factor, we can perform a similar analysis as in the one-loop case. However, we will see that this requires a more involved subtraction of the IR divergences. This will be the topic of the next section.

\section{Two-loop dilatation operator and remainder function}

In the one-loop case, the UV divergences stem from the bubble integrals alone. Therefore, the one-loop renormalisation constant can be read off directly from the coefficient of these integrals. This is no longer true for two-loop form factors, since the two-loop integrals in general contain a mixing of IR and UV divergences. However, IR divergences have a well-understood universal structure [37-40]. This allows us to subtract the IR divergences systematically using the BDS ansatz [41, 42].

Similar to the one-loop case (2.23), the two-loop renormalised form factor is given by

$$
\underline{\underline{I}}^{(2)}=\mathcal{I}^{(2)}+\mathcal{I}^{(1)} \mathcal{Z}^{(1)}+\mathcal{Z}^{(2)},
$$

where

$$
\mathcal{Z}^{(2)}=\sum_{i=1}^{L}\left(\mathcal{Z}_{i i+1 i+2}^{(2)}+\frac{1}{2} \sum_{j=i+2}^{L+i-2} \mathcal{Z}_{i i+1}^{(1)} \mathcal{Z}_{j j+1}^{(1)}\right) .
$$

Applying the BDS ansatz [41, 42] to the renormalised form factors, we obtain a finite two-loop remainder function:

$$
\mathcal{R}^{(2)}=\underline{\underline{I}}^{(2)}(\varepsilon)-\frac{1}{2}\left(\underline{\mathcal{I}}^{(1)}(\varepsilon)\right)^{2}-f^{(2)}(\varepsilon) \underline{\mathcal{I}}^{(1)}(2 \varepsilon)+\mathcal{O}(\varepsilon),
$$

where

$$
f^{(2)}(\varepsilon)=-2 \zeta_{2}-2 \zeta_{3} \varepsilon-2 \zeta_{4} \varepsilon^{2} .
$$

At two-loop order, connected interactions involve at most three fields of the composite operator, which have to be adjacent at the planar level. Hence, both the remainder function and the dilatation operator can be written in terms of densities that act only on triples of neighbouring sites at a time and are summed over all $L$ insertion points. For each triple of neighbouring points, we define the variables

$$
u_{i}=\frac{s_{i i+1}}{s_{i i+1 i+2}}, \quad v_{i}=\frac{s_{i+1 i+2}}{s_{i i+1 i+2}}, \quad w_{i}=\frac{s_{i+2 i}}{s_{i i+1 i+2}},
$$


where

$$
s_{i i+1 i+2}=s_{i i+1}+s_{i+1 i+2}+s_{i+2 i}
$$

and cyclic identification $i \sim i+L$ is understood. These variables satisfy $u_{i}+v_{i}+w_{i}=1$. The remainder $\mathcal{R}^{(2)}$ can be written in terms of its density as

$$
\mathcal{R}^{(2)}=\sum_{i=1}^{L} R_{i i+1 i+2}^{(2)} .
$$

An important subtlety arises due to the fact that the composite operators are not necessarily eigenstates under renormalisation. This requires a careful treatment of the product of one-loop form factors in (3.3). As already mentioned, the renormalisation constant is a matrix (i.e. an operator) and so are the interactions. Hence, the one-loop product in (3.3) should be understood as a product of operators. This can be explicitly depicted by the following equation in terms of graphs:

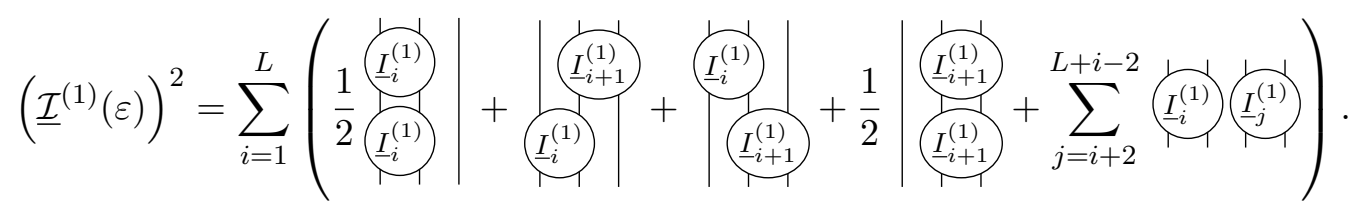

Note that the states corresponding to the internal lines are summed over as required for a product of operators. The prefactors of $\frac{1}{2}$ stem from distributing products of densities with effective range two equally between the first two and the last two sites.

The remainder density, which itself is an operator, can be similarly expressed by the following graph equation:

$$
\begin{aligned}
& R_{i i+1 i+2}^{(2)}=
\end{aligned}
$$

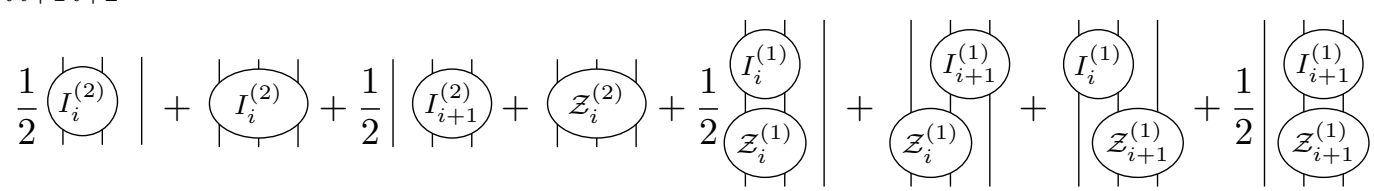

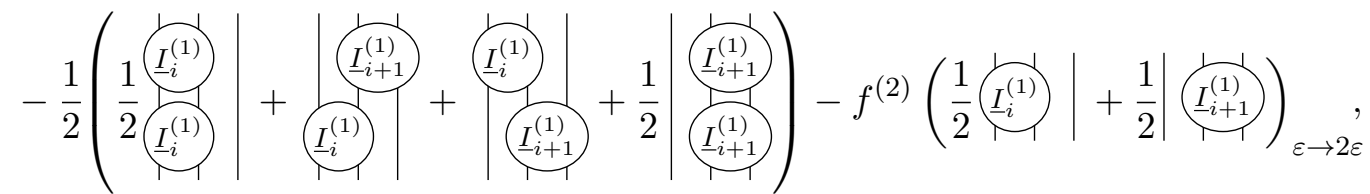

where we have depicted the two-loop renormalisation constant density $\mathcal{Z}_{i i+1 i+2}^{(2)}$ analogously to $I_{i i+1 i+2}^{(2)}$. Requiring that this remainder density is finite allows us to fix the two-loop renormalisation constant density.

The integrals occurring in the two-loop result can be reduced to master integrals via IBP reduction, e.g. as implemented in the Mathematica package LiteRed [60]. The resulting master integrals can be found in [61]. 


\subsection{Renormalisation constant and dilatation operator}

From the requirement that the two-loop renormalisation constant densities have to cancel all divergences in (3.9), we find

$$
\begin{array}{lll}
\left(\mathcal{Z}_{i}^{(2)}\right)_{X X X}^{X X X}=0, & \left(\mathcal{Z}_{i}^{(2)}\right)_{X Y X}^{X Y X}=+\frac{2}{\varepsilon^{2}}-\frac{2}{\varepsilon}, & \left(\mathcal{Z}_{i}^{(2)}\right)_{X X Y}^{X X Y}=+\frac{1}{2 \varepsilon^{2}}-\frac{1}{2 \varepsilon} \\
\left(\mathcal{Z}_{i}^{(2)}\right)_{X Y X}^{X X Y}=-\frac{1}{\varepsilon^{2}}+\frac{1}{\varepsilon}, & \left(\mathcal{Z}_{i}^{(2)}\right)_{X X Y}^{X Y X}=-\frac{1}{\varepsilon^{2}}+\frac{1}{\varepsilon}, & \left(\mathcal{Z}_{i}^{(2)}\right)_{X X Y}^{Y X X}=+\frac{1}{2 \varepsilon^{2}}-\frac{1}{2 \varepsilon}
\end{array}
$$

Alternatively, this can be written in the operatorial form ${ }^{13}$

$$
\mathcal{Z}_{i i+1 i+2}^{(2)}=\frac{1}{2}\left(\frac{1}{\varepsilon^{2}}-\frac{1}{\varepsilon}\right)\left(\mathbb{P}_{i i+1} \mathbb{P}_{i+1 i+2}+\mathbb{P}_{i+1 i+2} \mathbb{P}_{i i+1}-3 \mathbb{P}_{i i+1}-3 \mathbb{P}_{i+1 i+2}+4\right) .
$$

Using (1.3), we have for the two-loop dilatation operator

$$
\mathfrak{D}^{(2)}=4 \varepsilon\left(\mathcal{Z}^{(2)}-\frac{1}{2}\left(\mathcal{Z}^{(1)}\right)^{2}\right)
$$

where $\left(\mathcal{Z}^{(1)}\right)^{2}$ should be understood as a product of operators. For example,

$$
\begin{aligned}
\left(\mathfrak{D}_{i}^{(2)}\right)_{X X Y}^{X Y X}= & 4 \varepsilon\left(\left(\mathcal{Z}_{i}^{(2)}\right)_{X X Y}^{X Y X}-\frac{1}{2}\left(\mathcal{Z}_{i+1}^{(1)}\right)_{X Y}^{Y X}\left(\mathcal{Z}_{i}^{(1)}\right)_{X X}^{X X}-\frac{1}{2}\left(\mathcal{Z}_{i}^{(1)}\right)_{X Y}^{X Y}\left(\mathcal{Z}_{i+1}^{(1)}\right)_{X Y}^{Y X}\right. \\
& \left.-\frac{1}{4}\left(\mathcal{Z}_{i+1}^{(1)}\right)_{X Y}^{Y X}\left(\mathcal{Z}_{i+1}^{(1)}\right)_{X Y}^{X Y}-\frac{1}{4}\left(\mathcal{Z}_{i+1}^{(1)}\right)_{Y X}^{Y X}\left(\mathcal{Z}_{i+1}^{(1)}\right)_{X Y}^{Y X}\right)=4 .
\end{aligned}
$$

In total, we have

$$
\begin{array}{lll}
\left(\mathfrak{D}_{i}^{(2)}\right)_{X X X}^{X X X}=0, & \left(\mathfrak{D}_{i}^{(2)}\right)_{X Y X}^{X Y X}=-8, & \left(\mathfrak{D}_{i}^{(2)}\right)_{X X Y}^{X X Y}=-2, \\
\left(\mathfrak{D}_{i}^{(2)}\right)_{X Y X}^{X X Y}=4, & \left(\mathfrak{D}_{i}^{(2)}\right)_{X X Y}^{X Y X}=4, & \left(\mathfrak{D}_{i}^{(2)}\right)_{X X Y}^{Y X X}=-2,
\end{array}
$$

which agrees exactly with the known result [33]

$$
\mathfrak{D}_{i i+1 i+2}^{(2)}=-2\left(\mathbb{P}_{i i+1} \mathbb{P}_{i+1 i+2}+\mathbb{P}_{i+1 i+2} \mathbb{P}_{i i+1}-3 \mathbb{P}_{i i+1}-3 \mathbb{P}_{i+1 i+2}+4\right) .
$$

\subsection{Finite remainders}

Next, we calculate the finite remainder densities. The remainder densities fulfil analogous relations to $(2.35)$ :

$$
\begin{aligned}
& \left(R_{i}^{(2)}\right)_{X X X}^{Y X X}+\left(R_{i}^{(2)}\right)_{X X Y}^{X Y X}+\left(R_{i}^{(2)}\right)_{X X Y}^{X X Y}=\left(R_{i}^{(2)}\right)_{X X X}^{X X X}, \\
& \left(R_{i}^{(2)}\right)_{X Y X}^{X Y X}+\left(R_{i}^{(2)}\right)_{X Y X}^{Y X X}+\left(R_{i}^{(2)}\right)_{X Y X}^{X X Y}=\left(R_{i}^{(2)}\right)_{X X X}^{X X X}, \\
& \left(R_{i}^{(2)}\right)_{X X Y}^{X Y X}+\left(R_{i}^{(2)}\right)_{X X Y}^{Y X X}=\left(R_{i}^{(2)}\right)_{X Y X}^{X X Y}+\left(R_{i}^{(2)}\right)_{Y X X}^{X X Y}
\end{aligned}
$$

\footnotetext{
${ }^{13}$ Note that the coefficient of the simple pole coincides with the one of the double pole up to a sign. This is a consequence of the fact that at two loops only one Feynman integral with overall UV divergence occurs in the $\mathrm{SU}(2)$ sector when using a manifestly IR finite formulation, and it does not hold for general operators; cf. [34].
} 
These are equally a consequence of $\mathrm{SU}(2)$ symmetry and can be derived from

$$
\left[\mathfrak{J}^{A}, \mathcal{R}^{(2)}\right]=0
$$

which is a consequence of (2.19) and (2.36). Combining (3.16) with the symmetry under the exchange of $X \leftrightarrow Y$ and the reversion of the order of the fields, we can express all remainder densities in terms of $\left(R_{i}^{(2)}\right)_{X X X}^{X X X},\left(R_{i}^{(2)}\right)_{X X Y}^{X Y X}$ and $\left(R_{i}^{(2)}\right)_{X X Y}^{Y X X}$. Hence, it is enough to consider these three cases.

The remainder density $\left(R_{i}^{(2)}\right)_{X X X}^{X X X}$ was already studied in [14] and is of homogeneous transcendentality four:

$$
\left(R_{i}^{(2)}\right)_{X X X}^{X X X}=\left.\left(R_{i}^{(2)}\right)_{X X X X}^{X X X}\right|_{4}
$$

which is given explicitly as

$$
\begin{aligned}
&\left.\left(R_{i}^{(2)}\right)_{X X X}^{X X X}\right|_{4} \\
&=-\operatorname{Li}_{4}\left(1-u_{i}\right)-\operatorname{Li}_{4}\left(u_{i}\right)+\operatorname{Li}_{4}\left(\frac{u_{i}-1}{u_{i}}\right)-\log \left(\frac{1-u_{i}}{w_{i}}\right)\left[\operatorname{Li}_{3}\left(\frac{u_{i}-1}{u_{i}}\right)-\operatorname{Li}_{3}\left(1-u_{i}\right)\right] \\
&-\log \left(u_{i}\right)\left[\operatorname{Li}_{3}\left(\frac{v_{i}}{1-u_{i}}\right)+\operatorname{Li}_{3}\left(-\frac{w_{i}}{v_{i}}\right)+\operatorname{Li} 3\left(\frac{v_{i}-1}{v_{i}}\right)-\frac{1}{3} \log ^{3}\left(v_{i}\right)-\frac{1}{3} \log ^{3}\left(1-u_{i}\right)\right] \\
&-\operatorname{Li}_{2}\left(\frac{u_{i}-1}{u_{i}}\right) \operatorname{Li}_{2}\left(\frac{v_{i}}{1-u_{i}}\right)+\operatorname{Li}_{2}\left(u_{i}\right)\left[\log \left(\frac{1-u_{i}}{w_{i}}\right) \log \left(v_{i}\right)+\frac{1}{2} \log ^{2}\left(\frac{1-u_{i}}{w_{i}}\right)\right] \\
&+\frac{1}{24} \log ^{4}\left(u_{i}\right)-\frac{1}{8} \log ^{2}\left(u_{i}\right) \log ^{2}\left(v_{i}\right)-\frac{1}{2} \log ^{2}\left(1-u_{i}\right) \log \left(u_{i}\right) \log \left(\frac{w_{i}}{v_{i}}\right) \\
&-\frac{1}{2} \log \left(1-u_{i}\right) \log ^{2}\left(u_{i}\right) \log \left(v_{i}\right)-\frac{1}{6} \log ^{3}\left(u_{i}\right) \log \left(w_{i}\right) \\
&-\zeta_{2}\left[\log \left(u_{i}\right) \log \left(\frac{1-v_{i}}{v_{i}}\right)+\frac{1}{2} \log ^{2}\left(\frac{1-u_{i}}{w_{i}}\right)-\frac{1}{2} \log ^{2}\left(u_{i}\right)\right] \\
&+\zeta_{3} \log \left(u_{i}\right)+\frac{\zeta_{4}}{2}+G\left(\left\{1-u_{i}, 1-u_{i}, 1,0\right\}, v_{i}\right)+\left(u_{i} \leftrightarrow v_{i}\right) .
\end{aligned}
$$

Here, the Goncharov polylogarithm in the last line is the only piece that cannot be written in terms of classical polylogarithms. This relatively compact expression was obtained using the symbol techniques $[62,63]$. The corresponding symbol is given by $[14]^{14}$

$$
\begin{aligned}
\mathcal{S}\left(\left.\left(R_{i}^{(2)}\right)_{X X X X}^{X X X}\right|_{4}\right)= & -u_{i} \otimes\left(1-u_{i}\right) \otimes\left[\frac{u_{i}-1}{u_{i}} \otimes \frac{v_{i}}{w_{i}}+\frac{v_{i}}{w_{i}} \otimes \frac{w_{i}^{2}}{u_{i} v_{i}}\right]-u_{i} \otimes u_{i} \otimes \frac{1-u_{i}}{v_{i}} \otimes \frac{w_{i}}{v_{i}} \\
& -u_{i} \otimes v_{i} \otimes \frac{v_{i}}{w_{i}} \otimes \frac{u_{i}}{w_{i}}-u_{i} \otimes v_{i} \otimes \frac{u_{i}}{w_{i}} \otimes \frac{v_{i}}{w_{i}}+\left(u_{i} \leftrightarrow v_{i}\right) .
\end{aligned}
$$

The remainder density $\left(R_{i}^{(2)}\right)_{X X Y}^{X Y X}$ is of mixed transcendentality with degree ranging from three to zero. Its contribution of degree three has the symbol

$$
\mathcal{S}\left(\left.\left(R_{i}^{(2)}\right)_{X X Y}^{X Y X}\right|_{3}\right)=v_{i} \otimes \frac{v_{i}}{1-v_{i}} \otimes \frac{u_{i}}{1-v_{i}}-v_{i} \otimes \frac{1-v_{i}}{u_{i}} \otimes \frac{v_{i}}{w_{i}}-u_{i} \otimes \frac{1-u_{i}}{v_{i}} \otimes \frac{v_{i}}{w_{i}} .
$$

\footnotetext{
${ }^{14}$ The symbols can be conveniently calculated using the Mathematica code [64].
} 
The full transcendentality-three part can be given as

$$
\begin{aligned}
\left.\left(R_{i}^{(2)}\right)_{X X Y}^{X Y X}\right|_{3}= & {\left[\operatorname{Li}_{3}\left(-\frac{u_{i}}{w_{i}}\right)-\log \left(u_{i}\right) \operatorname{Li}_{2}\left(\frac{v_{i}}{1-u_{i}}\right)+\frac{1}{2} \log \left(1-u_{i}\right) \log \left(u_{i}\right) \log \left(\frac{w_{i}^{2}}{1-u_{i}}\right)\right.} \\
& \left.-\frac{1}{2} \operatorname{Li}_{3}\left(-\frac{u_{i} v_{i}}{w_{i}}\right)-\frac{1}{2} \log \left(u_{i}\right) \log \left(v_{i}\right) \log \left(w_{i}\right)-\frac{1}{12} \log ^{3}\left(w_{i}\right)+\left(u_{i} \leftrightarrow v_{i}\right)\right] \\
& -\operatorname{Li}_{3}\left(1-v_{i}\right)+\operatorname{Li}_{3}\left(u_{i}\right)-\frac{1}{2} \log ^{2}\left(v_{i}\right) \log \left(\frac{1-v_{i}}{u_{i}}\right)+\frac{1}{6} \pi^{2} \log \left(\frac{v_{i}}{w_{i}}\right) \\
& -\frac{1}{6} \pi^{2} \log \left(-s_{i i+1 i+2}\right) .
\end{aligned}
$$

Together with the terms of lower transcendentality, we have

$$
\begin{aligned}
\left(R_{i}^{(2)}\right)_{X X Y}^{X Y X}= & \left.\left(R_{i}^{(2)}\right)_{X X Y}^{X Y X}\right|_{3}+\operatorname{Li}_{2}\left(1-u_{i}\right)+\operatorname{Li}_{2}\left(1-v_{i}\right) \\
& +\log \left(u_{i}\right) \log \left(v_{i}\right)-\frac{1}{2} \log \left(-s_{i+1 i+2}\right) \log \left(\frac{u_{i}}{v_{i}}\right)+2 \log \left(-s_{i i+1}\right)+\frac{\pi^{2}}{3}-7 .
\end{aligned}
$$

The final remainder density $\left(R_{i}^{(2)}\right)_{X X Y}^{Y X X}$ is of mixed transcendentality with degree ranging from two to zero. It reads

$$
\begin{aligned}
\left(R_{i}^{(2)}\right)_{X X Y}^{Y X X}= & \frac{1}{2} \log \left(-s_{i+1 i+2}\right) \log \left(\frac{u_{i}}{v_{i}}\right)-\operatorname{Li}_{2}\left(1-u_{i}\right)-\log \left(u_{i}\right) \log \left(v_{i}\right)+\frac{1}{2} \log ^{2}\left(v_{i}\right) \\
& +\log \left(-s_{i+1 i+2}\right)-2 \log \left(-s_{i i+1}\right)+\frac{7}{2} .
\end{aligned}
$$

Let us emphasise that, if non-vanishing, the transcendentality-four contribution is the same for all remainder function densities. Furthermore, there is only one transcendentalitythree function and two functions of transcendentality smaller or equal to two that contribute to the results in the $\mathrm{SU}(2)$ sector. Notably, the highest degree of transcendentality $t=4-s$ is directly related to the shuffling number $s$ of the respective remainder density, i.e. to the number indicating by how many legs the field flavours are shuffled. For instance, $\left(R_{i}^{(2)}\right)_{X X Y}^{X Y X}$ has shuffling number $s=1$ and maximal transcendentality degree $t=3$.

Interestingly, the rational pieces of the remainder function are connected to the dilatation operator as

$$
\mathfrak{D}_{i i+1 i+2}^{(2)}=-\left.\frac{4}{7} R_{i i+1 i+2}^{(2)}\right|_{0} .
$$

\section{Conclusion and outlook}

In this paper, we have calculated the two-loop minimal form factor for all operators in the $\mathrm{SU}(2)$ sector of planar $\mathcal{N}=4 \mathrm{SYM}$ theory via the on-shell method of unitarity. Moreover, we have extracted the corresponding two-loop remainder function and the two-loop dilatation operator from it. The results of this paper provide a solid stepping stone towards calculating the complete two-loop dilatation operator of $\mathcal{N}=4 \mathrm{SYM}$ theory. The employed method, however, is independent of the high symmetry of planar $\mathcal{N}=4 \mathrm{SYM}$ theory, in particular of its integrability, and it is thus also applicable to less symmetric theories. 
The $\mathrm{SU}(2)$ sector is the simplest closed sector of the theory whose operators do not renormalise diagonally. It is hence well suited to study the occurrence of operator mixing and the dilatation operator. Due to the on-shell nature of the external fields, the divergences of the form factors are a combination of UV and IR divergences. We have disentangled the UV divergences from the IR divergences using the BDS ansatz and the universality of the latter. Because of the operator mixing, we needed to promote the interactions to operators and the iterative structure of the BDS ansatz to an operatorial form as well. From the UV divergences, we have determined the renormalisation constants and the dilatation operator.

In contrast to the BPS case, the two-loop remainders of non-protected operators in the $\mathrm{SU}(2)$ sector do not exhibit maximal uniform transcendentality. However, their maximally transcendental part coincides with the remainder of the BPS vacuum computed in [14]. These results and further evidence in other sectors lead us to conjecture that the twoloop remainder of every minimal form factor has the same degree-four part as the BPS one. Moreover, the two-loop remainder of the three-point form factor of every length-two operator should agree with the corresponding BPS remainder found in [8]. It would be interesting to check our conjecture about this universality for a wider class of operators, or even to prove it. ${ }^{15}$

Another observation is that the maximal transcendentality of the various remainders is related to their shuffling number, i.e. to the number indicating by how many places the field flavours are shuffled. It would be interesting to explore this pattern in larger sectors and at higher loops, where more complicated interactions contribute.

Soft or collinear limits of scattering amplitudes or form factors are typically given by lower-point amplitudes or form factors multiplied by a universal function. Thus, they provide important constraints and sometimes even allow to bootstrap the full function under consideration. As already observed in [14] for the BPS case, soft and collinear limits of minimal form factors also do not vanish for the cases considered here. This may be surprising since a priori there is no physical interpretation for this limit because these form factors correspond to the minimal physical configuration. Actually, similar questions also appear at the amplitude level. For example, taking certain soft or collinear limits of the sixscalar amplitudes given in appendix A does not generate any physical amplitude, though the limit is non-zero. Via unitarity cuts, this consideration on the level of amplitudes affects the limits of minimal form factors. It would be interesting to understand this point better, and to see if one can obtain the soft or collinear limit without computing the full quantity.

We have seen that the two-loop form factors, as well as the remainder functions and dilatation operator derived from them, obey Ward identities induced by the underlying

\footnotetext{
${ }^{15}$ One may expect that the maximally transcendental functions stem from the Laurent expansion of the functions with the highest-order poles in $\varepsilon$, which originate from pure IR divergences. As the IR divergences are universal, the maximally transcendental part of the remainder should also be universal. However, one should be cautious about this argument: for example, the leading transcendental functions of one-loop QCD amplitudes do not match with those of $\mathcal{N}=4$ SYM theory, although their IR divergences coincide [65]. An improved argument might proceed by showing the universality of the so-called leading singularities which are closely related to the maximally transcendental functions in the dlog form, as studied for amplitudes in $\mathcal{N}=4$ SYM theory e.g. in [49].
} 
R-symmetry. Going beyond the SU(2) sector, the realisation of similar Ward identities following from other symmetries should be more involved due to corrections to the generators which are absent for $\mathrm{SU}(2)$, cf. examples of such corrections in the case of spin chains [66] or scattering amplitudes [58,67]. Capturing these extensions in the case of form factors is currently under investigation.

\section{Acknowledgments}

It is a pleasure to thank Andreas Brandhuber, Burkhard Eden, Jan Fokken, Gregory Korchemsky, Brenda Penante, Gabriele Travaglini and Christian Vergu for useful discussions. FL would like to thank Simon Caron-Huot for initial collaboration on a related project and for useful discussions. We thank the Marie Curie network GATIS (gatis.desy.eu) of the European Union's Seventh Framework Programme FP7/2007-2013/ under REA Grant Agreement No 317089 for support. MW dankt der Studienstiftung des deutschen Volkes für ein Promotionsförderstipendium. DN and GY are supported by a DFG grant in the framework of the SFB 647 "Raum-Zeit-Materie. Analytische und Geometrische Strukturen".

\section{A Six-point scalar amplitudes}

In this appendix, we provide all six-point amplitudes that are required in the unitarity computation of the two-loop form factors in the $\mathrm{SU}(2)$ sector. We use the short notation

$$
\left(A^{(0)}\right)_{Z_{6} Z_{5} Z_{4}}^{Z_{1} Z_{2} Z_{3}}=\left.A^{(0)}\left(1^{Z_{1}}, 2^{Z_{2}}, 3^{Z_{3}}, 4^{\bar{Z}_{4}}, 5^{\bar{Z}_{5}}, 6^{\bar{Z}_{6}}\right)\right|_{\eta_{i}^{A}=1},
$$

where, on the right-hand side, all momenta are taken to be outgoing and we have set all $\eta_{i}$ variables to 1.

The required amplitudes can be explicitly given in terms of Mandelstam variables as

$$
\begin{aligned}
& \left(A^{(0)}\right)_{Y X X}^{X X Y}=-\frac{1}{s_{234}}, \\
& \left(A^{(0)}\right)_{X X Y}^{Y X X}=-\frac{1}{s_{345}}, \\
& \left(A^{(0)}\right)_{X X X}^{X X X}=\frac{s_{23} s_{56}}{s_{16} s_{34} s_{234}}+\frac{s_{12} s_{45}}{s_{16} s_{34} s_{345}}-\frac{s_{123}}{s_{16} s_{34}}, \\
& \left(A^{(0)}\right)_{X X Y}^{X Y X}=\frac{s_{12}}{s_{16} s_{345}}+\frac{s_{56}}{s_{16} s_{234}}-\frac{1}{s_{16}}+\frac{1}{s_{345}}, \\
& \left(A^{(0)}\right)_{Y X X}^{X Y X}=\frac{s_{23}}{s_{34} s_{234}}+\frac{s_{45}}{s_{34} s_{345}}-\frac{1}{s_{34}}+\frac{1}{s_{234}}, \\
& \left(A^{(0)}\right)_{X Y X}^{X X Y}=\frac{s_{12}}{s_{16} s_{345}}+\frac{s_{56}}{s_{16} s_{234}}-\frac{1}{s_{16}}+\frac{1}{s_{234}}, \\
& \left(A^{(0)}\right)_{X Y X}^{Y X X}=\frac{s_{23}}{s_{34} s_{234}}+\frac{s_{45}}{s_{34} s_{345}}-\frac{1}{s_{34}}+\frac{1}{s_{345}}, \\
& \left(A^{(0)}\right)_{X X Y}^{X X Y}=-\frac{s_{23} s_{56}}{s_{16} s_{34} s_{234}}-\frac{s_{12} s_{45}}{s_{16} s_{34} s_{345}}+\frac{s_{123}}{s_{16} s_{34}}-\frac{s_{12}}{s_{16} s_{345}}-\frac{s_{56}}{s_{16} s_{234}}+\frac{1}{s_{16}}, \\
& \left(A^{(0)}\right)_{Y X X}^{Y X X}=-\frac{s_{23} s_{56}}{s_{16} s_{34} s_{234}}-\frac{s_{12} s_{45}}{s_{16} s_{34} s_{345}}+\frac{s_{123}}{s_{16} s_{34}}-\frac{s_{23}}{s_{34} s_{234}}-\frac{s_{45}}{s_{34} s_{345}}+\frac{1}{s_{34}},
\end{aligned}
$$




$$
\begin{aligned}
\left(A^{(0)}\right)_{X Y X}^{X Y X}= & -\frac{s_{23} s_{56}}{s_{16} s_{34} s_{234}}-\frac{s_{12} s_{45}}{s_{16} s_{34} s_{345}}+\frac{s_{123}}{s_{16} s_{34}} \\
& -\frac{s_{12}}{s_{16} s_{345}}-\frac{s_{56}}{s_{16} s_{234}}+\frac{1}{s_{16}}-\frac{1}{s_{345}}-\frac{s_{23}}{s_{34} s_{234}}-\frac{s_{45}}{s_{34} s_{345}}+\frac{1}{s_{34}}-\frac{1}{s_{234}}
\end{aligned}
$$

where all poles are physical. ${ }^{16}$ Through the triple cut shown in figure $2 \mathrm{~d}$, each term in (A.2) is exactly mapped to one graph in table 3 .

It is easy to find various relations among these amplitudes, such as

$$
\begin{aligned}
& \left(A^{(0)}\right)_{X X Y}^{Y X X}+\left(A^{(0)}\right)_{X X Y}^{X Y X}+\left(A^{(0)}\right)_{X X Y}^{X X Y}=-\left(A^{(0)}\right)_{X X X}^{X X X}, \\
& \left(A^{(0)}\right)_{X Y X}^{X Y X}+\left(A^{(0)}\right)_{X Y X}^{Y X X}+\left(A^{(0)}\right)_{X Y X}^{X X Y}=-\left(A^{(0)}\right)_{X X X}^{X X X}, \\
& \left(A^{(0)}\right)_{X X Y}^{X Y X}+\left(A^{(0)}\right)_{X X Y}^{Y X X}=\left(A^{(0)}\right)_{X Y X}^{X X Y}+\left(A^{(0)}\right)_{Y X X}^{X X Y} .
\end{aligned}
$$

These are the counterparts of (2.35) and nothing but supersymmetric Ward identities (SWI) for amplitudes [68]. ${ }^{17}$

Open Access. This article is distributed under the terms of the Creative Commons Attribution License (CC-BY 4.0), which permits any use, distribution and reproduction in any medium, provided the original author(s) and source are credited.

\section{References}

[1] W.L. van Neerven, Infrared Behavior of On-shell Form-factors in a $\mathcal{N}=4$ Supersymmetric Yang-Mills Field Theory, Z. Phys. C 30 (1986) 595 [INSPIRE].

[2] A. Brandhuber, B. Spence, G. Travaglini and G. Yang, Form Factors in $\mathcal{N}=4$ Super Yang-Mills and Periodic Wilson Loops, JHEP 01 (2011) 134 [arXiv:1011.1899] [INSPIRE].

[3] L.V. Bork, D.I. Kazakov and G.S. Vartanov, On form factors in $\mathcal{N}=4 S Y M$, JHEP 02 (2011) 063 [arXiv: 1011.2440] [INSPIRE].

[4] A. Brandhuber, O. Gurdogan, R. Mooney, G. Travaglini and G. Yang, Harmony of Super Form Factors, JHEP 10 (2011) 046 [arXiv:1107.5067] [inSPIRE].

[5] L.V. Bork, D.I. Kazakov and G.S. Vartanov, On MHV Form Factors in Superspace for $\mathcal{N}=4$ SYM Theory, JHEP 10 (2011) 133 [arXiv:1107.5551] [INSPIRE].

[6] J.M. Henn, S. Moch and S.G. Naculich, Form factors and scattering amplitudes in $\mathcal{N}=4$ SYM in dimensional and massive regularizations, JHEP 12 (2011) 024 [arXiv:1109.5057] [INSPIRE].

[7] T. Gehrmann, J.M. Henn and T. Huber, The three-loop form factor in $\mathcal{N}=4$ super Yang-Mills, JHEP 03 (2012) 101 [arXiv:1112.4524] [INSPIRE].

[8] A. Brandhuber, G. Travaglini and G. Yang, Analytic two-loop form factors in $\mathcal{N}=4 S Y M$, JHEP 05 (2012) 082 [arXiv: 1201.4170] [INSPIRE].

[9] L.V. Bork, On NMHV form factors in $\mathcal{N}=4 S Y M$ theory from generalized unitarity, JHEP 01 (2013) 049 [arXiv: 1203.2596] [INSPIRE].

\footnotetext{
${ }^{16}$ The BCFW recursion relation or MHV rule methods directly give results in a much more complicated form, usually involving spurious poles. We have checked that these different methods give results equivalent to $($ A.2).

${ }^{17}$ The minus sign in front of $\left(A^{(0)}\right)_{X X X}^{X X X}$ is due to the convention for the fermionic $\eta$ variable in amplitudes.
} 
[10] O.T. Engelund and R. Roiban, Correlation functions of local composite operators from generalized unitarity, JHEP 03 (2013) 172 [arXiv:1209.0227] [INSPIRE].

[11] H. Johansson, D.A. Kosower and K.J. Larsen, Two-Loop Maximal Unitarity with External Masses, Phys. Rev. D 87 (2013) 025030 [arXiv:1208.1754] [InSPIRE].

[12] R.H. Boels, B.A. Kniehl, O.V. Tarasov and G. Yang, Color-kinematic Duality for Form Factors, JHEP 02 (2013) 063 [arXiv:1211.7028] [INSPIRE].

[13] B. Penante, B. Spence, G. Travaglini and C. Wen, On super form factors of half-BPS operators in $\mathcal{N}=4$ super Yang-Mills, JHEP 04 (2014) 083 [arXiv:1402.1300] [INSPIRE].

[14] A. Brandhuber, B. Penante, G. Travaglini and C. Wen, The last of the simple remainders, JHEP 08 (2014) 100 [arXiv: 1406.1443] [INSPIRE].

[15] L.V. Bork, On form factors in $\mathcal{N}=4$ SYM theory and polytopes, JHEP 12 (2014) 111 [arXiv: 1407.5568] [INSPIRE].

[16] M. Wilhelm, Amplitudes, Form Factors and the Dilatation Operator in $\mathcal{N}=4$ SYM Theory, JHEP 02 (2015) 149 [arXiv: 1410.6309] [INSPIRE].

[17] D. Nandan, C. Sieg, M. Wilhelm and G. Yang, Cutting through form factors and cross sections of non-protected operators in $\mathcal{N}=4$ SYM, JHEP 06 (2015) 156 [arXiv:1410.8485] [INSPIRE].

[18] L.F. Alday and J. Maldacena, Comments on gluon scattering amplitudes via AdS/CFT, JHEP 11 (2007) 068 [arXiv:0710.1060] [INSPIRE].

[19] J. Maldacena and A. Zhiboedov, Form factors at strong coupling via a Y-system, JHEP 11 (2010) 104 [arXiv:1009.1139] [INSPIRE].

[20] Z. Gao and G. Yang, Y-system for form factors at strong coupling in $A d S_{5}$ and with multi-operator insertions in $A d S_{3}$, JHEP 06 (2013) 105 [arXiv:1303.2668] [INSPIRE].

[21] N. Beisert, The complete one loop dilatation operator of $\mathcal{N}=4$ super Yang-Mills theory, Nucl. Phys. B 676 (2004) 3 [hep-th/0307015] [INSPIRE].

[22] B.I. Zwiebel, From Scattering Amplitudes to the Dilatation Generator in $\mathcal{N}=4 S Y M$, J. Phys. A 45 (2012) 115401 [arXiv:1111.0083] [inSPIRE].

[23] Z. Bern, L.J. Dixon, D.C. Dunbar and D.A. Kosower, One loop n-point gauge theory amplitudes, unitarity and collinear limits, Nucl. Phys. B 425 (1994) 217 [hep-ph/9403226] [INSPIRE].

[24] Z. Bern, L.J. Dixon, D.C. Dunbar and D.A. Kosower, Fusing gauge theory tree amplitudes into loop amplitudes, Nucl. Phys. B 435 (1995) 59 [hep-ph/9409265] [INSPIRE].

[25] R. Britto, F. Cachazo and B. Feng, Generalized unitarity and one-loop amplitudes in $\mathcal{N}=4$ super-Yang-Mills, Nucl. Phys. B 725 (2005) 275 [hep-th/0412103] [INSPIRE].

[26] A. Brandhuber, B. Penante, G. Travaglini and D. Young, Integrability and unitarity, JHEP 05 (2015) 005 [arXiv: 1502.06627] [INSPIRE].

[27] O.T. Engelund, Lagrangian Insertion in the Light-Like Limit and the Super-Correlators/ Super-Amplitudes Duality, arXiv:1502.01934 [INSPIRE].

[28] L. Koster, V. Mitev and M. Staudacher, A Twistorial Approach to Integrability in $\mathcal{N}=4$ SYM, Fortsch. Phys. 63 (2015) 142 [arXiv:1410.6310] [InSPIRE].

[29] D. Chicherin, R. Doobary, B. Eden, P. Heslop, G.P. Korchemsky, L. Mason and E. Sokatchev, Correlation functions of the chiral stress-tensor multiplet in $\mathcal{N}=4 S Y M$, JHEP 06 (2015) 198 [arXiv:1412.8718] [INSPIRE]. 
[30] A. Brandhuber, B. Penante, G. Travaglini and D. Young, Integrability and MHV diagrams in $\mathcal{N}=4$ supersymmetric Yang-Mills theory, Phys. Rev. Lett. 114 (2015) 071602 [arXiv: 1412.1019] [INSPIRE].

[31] N. Beisert et al., Review of AdS/CFT Integrability: An Overview, Lett. Math. Phys. 99 (2012) 3 [arXiv: 1012.3982] [InSPIRE].

[32] J.A. Minahan and K. Zarembo, The Bethe ansatz for $\mathcal{N}=4$ super Yang-Mills, JHEP 03 (2003) 013 [hep-th/0212208] [INSPIRE].

[33] N. Beisert, C. Kristjansen and M. Staudacher, The Dilatation operator of conformal $\mathcal{N}=4$ super Yang-Mills theory, Nucl. Phys. B 664 (2003) 131 [hep-th/0303060] [INSPIRE].

[34] C. Sieg, Superspace computation of the three-loop dilatation operator of $\mathcal{N}=4$ SYM theory, Phys. Rev. D 84 (2011) 045014 [arXiv: 1008.3351] [INSPIRE].

[35] T. Bargheer, N. Beisert and F. Loebbert, Boosting Nearest-Neighbour to Long-Range Integrable Spin Chains, J. Stat. Mech. 0811 (2008) L11001 [arXiv:0807.5081] [INSPIRE].

[36] T. Bargheer, N. Beisert and F. Loebbert, Long-Range Deformations for Integrable Spin Chains, J. Phys. A 42 (2009) 285205 [arXiv:0902.0956] [INSPIRE].

[37] A.H. Mueller, On the Asymptotic Behavior of the Sudakov Form-factor, Phys. Rev. D 20 (1979) 2037 [INSPIRE].

[38] J.C. Collins, Algorithm to Compute Corrections to the Sudakov Form-factor, Phys. Rev. D 22 (1980) 1478 [inSPIRE].

[39] A. Sen, Asymptotic Behavior of the Sudakov Form-Factor in QCD, Phys. Rev. D 24 (1981) 3281 [inSPIRE].

[40] L. Magnea and G.F. Sterman, Analytic continuation of the Sudakov form-factor in QCD, Phys. Rev. D 42 (1990) 4222 [INSPIRE].

[41] C. Anastasiou, Z. Bern, L.J. Dixon and D.A. Kosower, Planar amplitudes in maximally supersymmetric Yang-Mills theory, Phys. Rev. Lett. 91 (2003) 251602 [hep-th/0309040] [INSPIRE].

[42] Z. Bern, L.J. Dixon and V.A. Smirnov, Iteration of planar amplitudes in maximally supersymmetric Yang-Mills theory at three loops and beyond, Phys. Rev. D 72 (2005) 085001 [hep-th/0505205] [inSPIRE].

[43] S. Catani, The Singular behavior of QCD amplitudes at two loop order, Phys. Lett. B 427 (1998) 161 [hep-ph/9802439] [INSPIRE].

[44] G.F. Sterman and M.E. Tejeda-Yeomans, Multiloop amplitudes and resummation, Phys. Lett. B 552 (2003) 48 [hep-ph/0210130] [INSPIRE].

[45] J. Bartels, L.N. Lipatov and A. Sabio Vera, BFKL Pomeron, Reggeized gluons and Bern-Dixon-Smirnov amplitudes, Phys. Rev. D 80 (2009) 045002 [arXiv:0802.2065] [INSPIRE].

[46] Z. Bern et al., The Two-Loop Six-Gluon MHV Amplitude in Maximally Supersymmetric Yang-Mills Theory, Phys. Rev. D 78 (2008) 045007 [arXiv:0803.1465] [InSPIRE].

[47] J.M. Drummond, J. Henn, G.P. Korchemsky and E. Sokatchev, Hexagon Wilson loop = six-gluon MHV amplitude, Nucl. Phys. B 815 (2009) 142 [arXiv:0803.1466] [INSPIRE].

[48] S. Caron-Huot and K.J. Larsen, Uniqueness of two-loop master contours, JHEP 10 (2012) 026 [arXiv:1205.0801] [InSPIRE].

[49] N. Arkani-Hamed, J.L. Bourjaily, F. Cachazo, A.B. Goncharov, A. Postnikov and J. Trnka, Scattering Amplitudes and the Positive Grassmannian, arXiv:1212.5605 [INSPIRE]. 
[50] D. Nandan, M.F. Paulos, M. Spradlin and A. Volovich, Star Integrals, Convolutions and Simplices, JHEP 05 (2013) 105 [arXiv: 1301.2500] [INSPIRE].

[51] A.V. Kotikov and L.N. Lipatov, DGLAP and BFKL evolution equations in the $\mathcal{N}=4$ supersymmetric gauge theory, hep-ph/0112346 [INSPIRE].

[52] A.V. Kotikov, L.N. Lipatov, A.I. Onishchenko and V.N. Velizhanin, Three loop universal anomalous dimension of the Wilson operators in $\mathcal{N}=4$ SUSY Yang-Mills model, Phys. Lett. B 595 (2004) 521 [Erratum ibid. B 632 (2006) 754] [hep-th/0404092] [INSPIRE].

[53] A.V. Kotikov and L.N. Lipatov, On the highest transcendentality in $\mathcal{N}=4$ SUSY, Nucl. Phys. B 769 (2007) 217 [hep-th/0611204] [INSPIRE].

[54] Y. Li, A. von Manteuffel, R.M. Schabinger and H.X. Zhu, Soft-virtual corrections to Higgs production at $N^{3} L O$, Phys. Rev. D 91 (2015) 036008 [arXiv:1412.2771] [INSPIRE].

[55] T. Gehrmann, M. Jaquier, E.W.N. Glover and A. Koukoutsakis, Two-Loop QCD Corrections to the Helicity Amplitudes for $H \rightarrow 3$ partons, JHEP 02 (2012) 056 [arXiv:1112.3554] [INSPIRE].

[56] A.V. Belitsky, S. Hohenegger, G.P. Korchemsky, E. Sokatchev and A. Zhiboedov, Energy-Energy Correlations in $\mathcal{N}=4$ Supersymmetric Yang-Mills Theory, Phys. Rev. Lett. 112 (2014) 071601 [arXiv:1311.6800] [INSPIRE].

[57] V.P. Nair, A Current Algebra for Some Gauge Theory Amplitudes, Phys. Lett. B 214 (1988) 215 [inSPIRE].

[58] T. Bargheer, N. Beisert, W. Galleas, F. Loebbert and T. McLoughlin, Exacting $\mathcal{N}=4$ Superconformal Symmetry, JHEP 11 (2009) 056 [arXiv:0905.3738] [INSPIRE].

[59] V.A. Smirnov, Springer Tracts in Modern Physics. Vol. 211: Evaluating Feynman integrals, Springer, Berlin U.K. (2004).

[60] R.N. Lee, LiteRed 1.4: a powerful tool for reduction of multiloop integrals, J. Phys. Conf. Ser. 523 (2014) 012059 [arXiv:1310.1145] [InSPIRE].

[61] T. Gehrmann and E. Remiddi, Two loop master integrals for $\gamma^{*} \rightarrow 3$ jets: The Planar topologies, Nucl. Phys. B 601 (2001) 248 [hep-ph/0008287] [INSPIRE].

[62] A.B. Goncharov, A simple construction of Grassmannian polylogarithms, arXiv:0908.2238 [INSPIRE].

[63] A.B. Goncharov, M. Spradlin, C. Vergu and A. Volovich, Classical Polylogarithms for Amplitudes and Wilson Loops, Phys. Rev. Lett. 105 (2010) 151605 [arXiv:1006.5703] [INSPIRE].

[64] C. Vergu, Lecture Notes for the Mathematica Summer School on Theoretical Physics, http://msstp.org/sites/default/files/Demo.nb (2011).

[65] Z. Bern and D.A. Kosower, The Computation of loop amplitudes in gauge theories, Nucl. Phys. B 379 (1992) 451 [INSPIRE].

[66] N. Beisert, The $\mathrm{SU}(2 \mid 3)$ dynamic spin chain, Nucl. Phys. B 682 (2004) 487 [hep-th/0310252] [INSPIRE].

[67] N. Beisert, J. Henn, T. McLoughlin and J. Plefka, One-Loop Superconformal and Yangian Symmetries of Scattering Amplitudes in $\mathcal{N}=4$ Super Yang-Mills, JHEP 04 (2010) 085 [arXiv: 1002.1733] [INSPIRE].

[68] M.T. Grisaru, H.N. Pendleton and P. van Nieuwenhuizen, Supergravity and the S Matrix, Phys. Rev. D 15 (1977) 996 [inSPIRE]. 